University of Montana

ScholarWorks at University of Montana

9-15-1999

\title{
Micromagnetics Simulation of Nanoshaped Iron Elements: Comparison with Experiment
}

NgocNga Dao

University of New Orleans

S. R. Homer

University of New Orleans

Scott L. Whittenburg

University of Montana - Missoula, scott.whittenburg@umontana.edu

Follow this and additional works at: https://scholarworks.umt.edu/chem_pubs

Part of the Biochemistry Commons, Chemistry Commons, and the Physics Commons Let us know how access to this document benefits you.

\section{Recommended Citation}

Dao, NgocNga; Homer, S. R.; and Whittenburg, Scott L., "Micromagnetics Simulation of Nanoshaped Iron Elements: Comparison with Experiment" (1999). Chemistry and Biochemistry Faculty Publications. 61. https://scholarworks.umt.edu/chem_pubs/61

This Article is brought to you for free and open access by the Chemistry and Biochemistry at ScholarWorks at University of Montana. It has been accepted for inclusion in Chemistry and Biochemistry Faculty Publications by an authorized administrator of ScholarWorks at University of Montana. For more information, please contact scholarworks@mso.umt.edu. 


\title{
Micromagnetics simulation of nanoshaped iron elements: Comparison with experiment
}

\author{
N. Dao, S. R. Homer, and S. L. Whittenburga) \\ Department of Chemistry and Advanced Materials Research Institute, University of New Orleans, \\ New Orleans, Louisiana 70148
}

(Received 3 March 1999; accepted for publication 10 June 1999)

\begin{abstract}
A micromagnetics simulation has been conducted on nanostructured magnetic elements of iron in order to investigate the effect of the shape of the element on magnetic properties, such as domain formation and hysteresis loops. These results are compared with recent experimental studies. The results display an impressive agreement with both the experimentally observed magnetic domains in individual particles as well as the shape of the hysteresis loops. The simulation results then explain features in the hysteresis loops in terms of vortice formation and motion. (c) 1999 American Institute of Physics. [S0021-8979(99)04018-9]
\end{abstract}

\section{INTRODUCTION}

There is currently great interest in nanostructured magnetic elements particularly in their application to magnetic memory, high density recording media, and magnetic field sensors. As these magnetic elements have decreased in size, their magnetic properties such as magnetic switching, coercivity, and hysteresis loops have begun to demonstrate unexpected behavior. For small elements, the shape of the element and the temperature both have a dramatic effect on the magnetic properties. ${ }^{1}$ The shape dependence of the magnetic properties on nanoelements has previously been shown both experimentally ${ }^{2,3}$ and computationally ${ }^{4}$ for $\mathrm{NiFe}$. In this project, we will examine the effect of the magnetic element for Fe thin films on magnetic domains and switching using micromagnetics simulations.

\section{PROCEDURE}

All micromagnetics simulations were performed using the OOMMF (Object Oriented MicroMagnetics Framework) code from NIST. This code is freeware and is obtainable over the internet ${ }^{5}$ with source code. It is supplied with a utility to build the executable on a variety of computer architectures and the graphical front end is written in Tcl/Tk so that OOMMF has a similar look-and-feel on a wide range of computer systems. Conventional terms for the various contributions to the local field and energy are included in the calculation. The exchange energy is computed via an eightneighbor dot product ${ }^{6}$ and the magnetostatic energy is calculated using a fast Fourier transform (FFT)-based scalar potential. ${ }^{7}$ The magnetization of the sample is computed as a function of time using damped Landau-Lifshitz-Gilbert equations of motion.

The magnetic properties of nanoshaped iron elements have been measured experimentally and recently reported. ${ }^{8}$ In this study, two different nanoshaped elements were studied; rectangular and needle shaped. An image of the elements is given in Fig. 1. Both elements were $0.5 \mu \mathrm{m}$ wide

a)Electronic mail: swhitten@uno.edu epitaxial (110) Fe. The length of the element is determined by the experimental dimensions of each element. It is roughly three times the width or $1.5 \mu \mathrm{m}$ for the rectangular elements and five times the width of $2.5 \mu \mathrm{m}$ for the needleshaped elements. The film thickness for all examples was 50 $\mathrm{nm}$. The rectangular elements had significant rounding of the corners. The magnetic properties and the magnetization reversal process were studied using magneto-optical Kerr effect (MOKE) and magnetic force microscopy (MFM). The hysteresis loops were measured on arrays of these nanoshaped elements where the elements were separated by four times their length and three times their width in order to minimize dipolar interactions between the elements. One feature of OOMMF is the ability to simulate unusual shaped elements by mapping the spins onto a graphics interchange format (GIF) (or other) image format. In this work, we scanned the pictures of the experimental nanoshaped elements and converted the scanned image into a GIF file. Thus, the simulations were performed on the same shaped elements as measured experimentally. For the hysteresis loops, the simulation was performed on a $2 \times 3$ array of elements. While the experimental loops were measured on a larger array of particles, and thus the variation in shape may be important, the simulation still accounts for some variation in shape and possible dipolar coupling between elements. For all simulations reported here, the cell size was taken as 10 $\mathrm{nm}$. This corresponds to $6 \times 10^{5}$ spins. As a test of the suitability of this cell size, for the longitudinal saturation of the rectangular element one simulation was conducted with a 5 $\mathrm{nm}$ cell size. Since the cell dimensions were doubled in each of the $x, y$, and $z$ directions this corresponds to an eight-fold increase in the number of spins or $4.8 \times 10^{6}$ spins. The domain pattern obtained in this simulation matched both the $10 \mathrm{~nm}$ simulation result as well as the experimental pattern. The larger cell size was used in further simulations including the computation of the hysteresis loops. The OOMMF standard iron parameters were used: $M_{s}=1700 \times 10^{3} \mathrm{~A} / \mathrm{m}$, $A=21 \times 10^{12} \mathrm{~J} / \mathrm{m}$, and $K_{1}=47.0 \times 10^{3} \mathrm{~J} / \mathrm{m}^{3}$. 

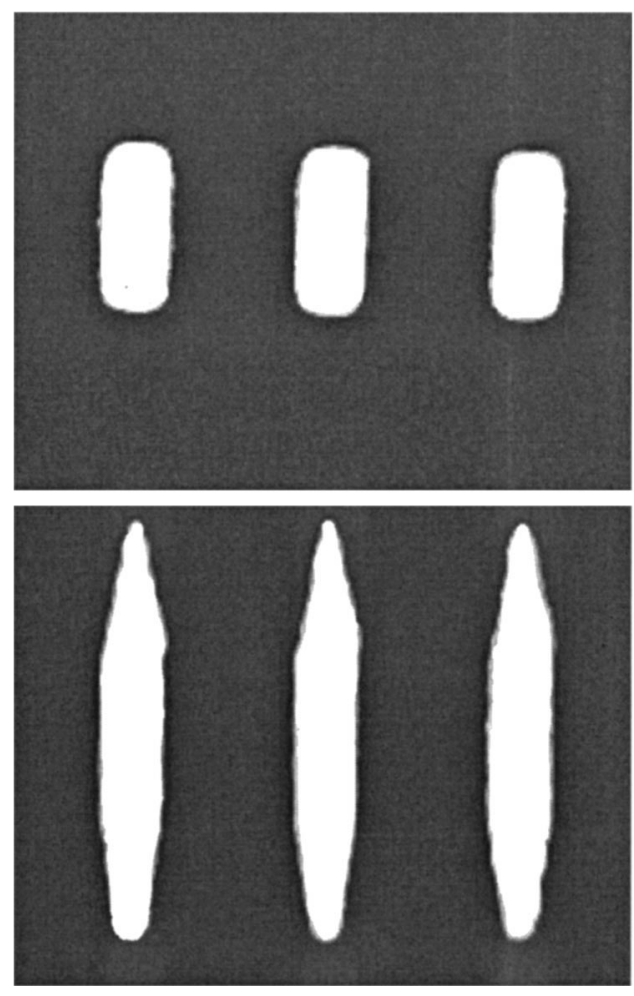

FIG. 1. SEM images of the rectangular and needle-shaped (110) Fe elements. The easy axis is (001) and is perpendicular to the long axis of the elements. Image adapted from Ref. 5.

\section{RESULTS}

Before attempting to compute the hysteresis loops, it is worthwhile computing the domain structure of individual elements. MFM images were obtained on single elements, both rectangular and needle shaped following application and removal of a saturating field. The saturating field was applied both longitudinal and transverse to the long axis of the element. The influence of particle shape and magnetic history on the domain structure is quite interesting. The experimentally measured domain structure is shown in Fig. 2, while the result of the micromagnetics simulation is given in Fig. 3. The agreement between the simulated MFM maps and the experimental ones is excellent. For rectangular elements following longitudinal saturation, the elements contain

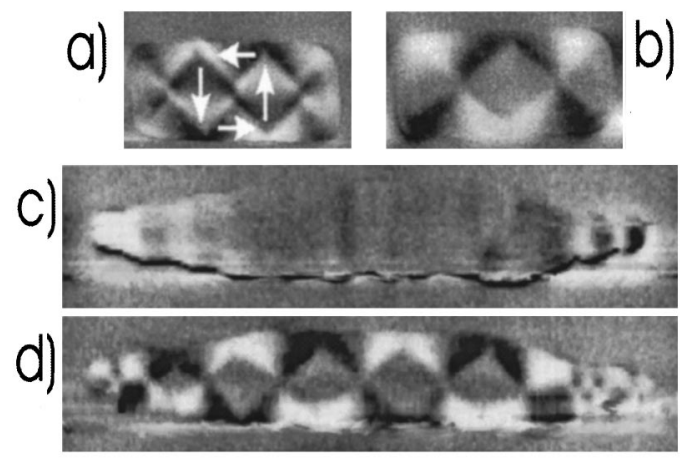

FIG. 2. MFM images at $H=0$ on rectangular and needle-shaped Fe elements following (a) and (c) longitudinal saturation and following (b) and (d) transverse magnetic saturation. Image adapted from Ref. 5. (a)

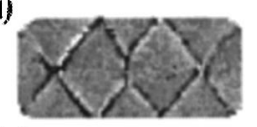

(c)

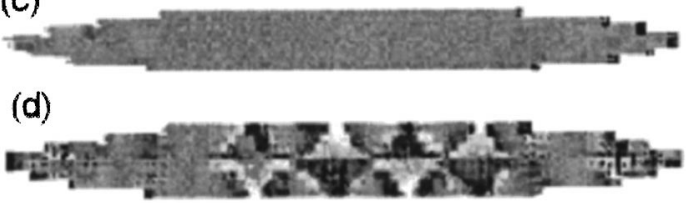

FIG. 3. Magnetic domain structure predicted from the micromagnetics simulation for the same conditions as in Fig. 2.

three vortices, while transverse saturation and field removal produces a domain structure with two vortices. For needleshaped elements, the domain structure is more interesting. For transverse saturation followed by field removal, the domain structure displays five major vortices aligned along the long axis of the needle. For longitudinal saturation, the needle-shaped element maintains a single magnetic domain in the fully saturated state even after the external field is removed. The picture of spins obtained from the micromagnetics simulation explains this difference. In the needleshaped element following longitudinal saturation, the magnetostatic energy is quite small due to the fact that a large percentage of spins is near the surface of the element and these spins are aligned along the edge of the element. Attempts to disrupt this arrangement, even in the absence of the externally applied magnetic field, require a large increase in the magnetostatic energy. In the rectangular elements, there is a smaller percentage of spins near the surface of the particle and, perhaps more importantly, the corner of the rectangle serves as a "seed" for the formation of a vortice. The spin reversal mechanism in the rectangular elements always proceeds via the formation of a vortice in the corner of the rectangle followed by motion of the vortice along the element edge.

We have also simulated the hysteresis loops of an array of rectangular and needle-shaped elements. For each shape, an array of $2 \times 3$ elements was chosen for the simulation with the separation between the elements chosen the same as the experimental parameters. Each hysteresis loop consisted of 200 field values and took approximately 1.5 days on a 300 $\mathrm{MHz}$ DEC alpha workstation to compute. For the rectangular elements, the hysteresis loops consisted of 200 points ranging from -500 to $+500 \mathrm{mT}$ with an increment of $10 \mathrm{mT}$ for both the forward and reverse loops. For the needle-shaped elements, the loops consisted of 200 points ranging from -1000 to $+1000 \mathrm{mT}$ with an increment of $20 \mathrm{mT}$ for both the forward and reverse loops. The experimental loops for both longitudinal and transverse applied fields for both rectangular and needle-shaped elements are given in Fig. 4. The corresponding simulated loops are given in Fig. 5. The correspondence between the shapes of the simulated and experimental hysteresis loops is remarkable and lends support to the use of the simulated loops in interpreting the experimental results.

The greatest value of the micromagnetics simulation is the ability to view changes in the magnetic domain structure 


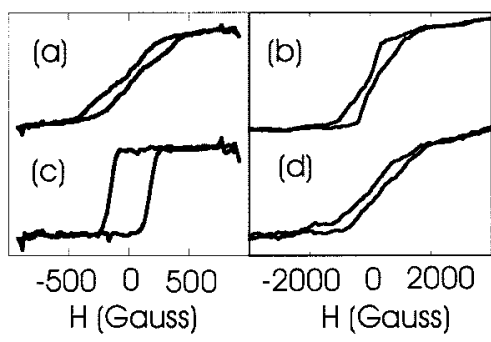

FIG. 4. Experimental MOKE hysteresis loops nanoshaped epitaxial (110) Fe elements. The applied field is applied longitudinally for (a) rectangular and (c) needle-shaped elements and transverse for (b) rectangular and (d) needle-shaped elements. Data taken from Ref. 5.

at each step in the hysteresis loop. From these calculations, we can begin to understand the magnetization reversal process. For both the rectangular and needle-shaped elements, the magnetization reversal begins with the formation of a vortice in a "corner" of the element. As can be seen in Fig. 1 , for the needle-shaped elements, one end of the element is more rounded than the other. The vortice always forms at the rounded end. The reversal process evolves with a twisting magnetization reversal through the middle of the element until suddenly the interior of the elements switches to align along the applied field. This occurs when the Zeeman energy is significantly larger than the sum of the exchange, anisot-
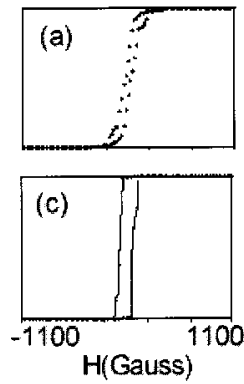
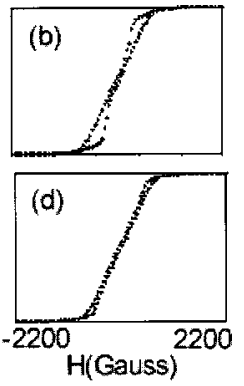

FIG. 5. Micromagnetics simulation result for the hysteresis of the $\mathrm{Fe}$ nanoshaped particle arrays. The labels correspond to Fig. 4. ropy, and magnetostatic energy. In the needle-shaped elements, this corresponds to a domain pattern where the vast majority of spins in the interior of the element are aligned along the applied field while a small "skin" of spins are pinned in the opposite direction by the magnetostatic energy. This state gives a lower magnetostatic energy at the expense of the exchange energy. While this skin appears to be a collapsed Neel wall, it would likely be resolved as a true Neel wall with crossties if simulated with a variational grid approach. ${ }^{9}$ As the applied field is increased further, the surface spins reorient to align along the applied field until, eventually, all spins are aligned along the applied field. The micromagnetics simulation explains the importance of the magnetostatic energy in shape of the hysteresis loops and in the apparent coercivity. Also, the simulations show that the tip shape of the nanoshaped elements is crucial in determining the magnetization reversal process.

\section{ACKNOWLEDGMENTS}

The authors wish to thank the support of DARPA via DOD/DARPA MDA972-97-1-0003. The authors also wish to thank Andy Kent for providing the images and hysteresis data from the experimental work on the nanoshaped iron elements.

${ }^{1}$ J. Zhu, Y. Zheng, and T. Fang, Proceedings of Spintronics '98, DARPA, Arlington, VA, 1998, p. 542.

${ }^{2}$ M. Ruhrig, B. Khamsehpour, K. J. Kirk, J. N. Chapman, P. Aitchison, S. McVitie, and C. D. W. Wilkinson, IEEE Trans. Magn. 32, 4452 (1996).

${ }^{3}$ K. J. Kirk, J. N. Chapman, and C. D. W. Wilkinson, Appl. Phys. Lett. 71, 539 (1997).

${ }^{4}$ T. Schrefl, J. Fidler, K. J. Kirk, and J. N. Chapman, IEEE Trans. Magn. 33, 4182 (1997)

${ }^{5}$ See math.nist.gov/oommf.

${ }^{6}$ M. J. Donahue and R. D. McMichael, Physica B 233, 272 (1997).

${ }^{7}$ D. V. Berkov, K. Ramstock, and A. Hubert, Phys. Status Solidi A 137, 207 (1993).

${ }^{8}$ J. Yu, U. Rudiger, L. Thomas, S. S. Parkin, and A. D. Kent, J. Appl. Phys. 85, 5501 (1999).

${ }^{9}$ M. J. Donahue, J. Appl. Phys. 83, 6491 (1998). 